\title{
El rol de la edad en el estado de ánimo asociado al rechazo parental de la homosexualidad*
}

Cómo citar este artículo:

Quiroga-Garza, A. y Gómez, A. A. (2021).

El rol de la edad en el estado de ánimo

asociado al rechazo parental de la

homosexualidad. Revista Latinoamericana

de Estudios de Familia, 13(2), 163-184.

https://doi.org/10.17151/rlef.2021.13.2.9

\author{
Angélica Quiroga-Garza* \\ Amaryllis A. Gómez-Rodríguez ${ }^{* * *}$
}

Recibido: 26 de agosto de 2020

Aprobado: 27 de abril de 2021

Resumen: Objetivo. En el presente estudio se buscó demostrar de qué manera se explica la depresión comúnmente asociada a la falta de aceptación personal y familiar de las personas homosexuales. Material y método. Investigación empírica cuantitativa, explicativa, de corte transversal. Participaron 354 personas, hombres y mujeres homosexuales mayores de edad en forma voluntaria y anónima. Se aplicaron la Depression Anxiety Stress Scale (DASS21), el Cuestionario de Aceptación Personal y Apoyo Familiar y un Cuestionario sociodemográfico diseñado para el estudio. Resultados. El hallazgo principal fue un efecto directo de la aceptación personal y familiar de la homosexualidad filial en la depresión mediada por la edad que indica que entre más edad tenga el hijo homosexual, tiende a desaparecer el impacto negativo del rechazo parental en su estado de ánimo. Conclusiones. Probado el deterioro emocional que ocasiona la no aceptación familiar que mantiene el ocultamiento de la homosexualidad filial, urge mayor educación sobre el tema a los padres, así como la implementación de políticas públicas que garanticen el reconocimiento, atención y respeto a sus derechos.

Palabras clave: homosexualidad, discriminación, heteronormatividad, depresión, familia, salud mental.

\footnotetext{
*Descripción. Investigación realizada por profesionales del Centro de Tratamiento e Investigación de la Ansiedad (CETIA) en Monterrey, México, en la necesidad de evidenciar el alto índice de ansiedad y depresión dentro de la comunidad LGBTTTIQ, su influencia en la dinámica familiar y la importancia de la inclusión familiar de un hijo con una orientación homosexual.

** Universidad de Monterrey. San Pedro Garza García, México. E-mail: angelica.quiroga@udem.edu.

(iD orcid.org/0000-0003-3991-2261. Google Scholar

* Universidad de Monterrey. San Pedro Garza García, México. E-mail: amaryllis.amanda@gmail.com.

(iD) orcid.org/0000-0003-4155-4571. Google Scholar
}

DOI: 10.17151/rlef.2021.13.2.9. 


\title{
The role of age in mood associated with parental rejection of homosexuality
}

\begin{abstract}
Objective. This study intended to demonstrate how depression commonly associated with the lack of personal and family acceptance of homosexual people is explained. Materials and methods. Quantitative, explanatory, cross-sectional empirical research. A total of 354 homosexual men and women of legal age participated voluntarily and anonymously. The Depression Anxiety Stress Scale (DASS-21), the Personal Acceptance and Family Support Questionnaire and a sociodemographic questionnaire designed for the study were applied. Results. The main finding was a direct effect of personal and family acceptance of filial homosexuality in agemediated depression, which indicates that the older the homosexual child is, the negative impact of parental rejection on his state of life tends to disappear. Conclusion. Having proven the emotional deterioration caused by family non-acceptance that maintains the concealment of filial homosexuality, greater education on this issue for parents is urgently needed as well as the implementation of public policies that guarantee the recognition, attention and respect of their rights.
\end{abstract}

Key words: homosexuality, discrimination, heteronormativity, depression, family, mental health.

\section{Introducción}

Cada vez son más los jóvenes miembros de la comunidad lésbico, gay, bisexual, transexual, travesti, transgénero, intersexual u otras identidades de género (LGBTI+) y de expresiones sexuales - formas en que se puede manifestar el comportamiento sexual sin connotación moral [bueno-malo] (Lizárraga, 2014)— que deciden compartir su identidad no heteronormada (Lipkin, 2018), sin embargo, al develarla con frecuencia se ven enfrentados al estigma y la discriminación. Así lo demuestran los resultados de la Encuesta Nacional sobre Discriminación (ENADIS) realizada en 2017 por el Instituto Nacional de Estadística, Geografía e Informática (INEGI, 2018a) en México. Casi 2'700.000 de las 84’008.180 personas encuestadas, declararon no ser heterosexuales, es decir, el 3,2\% de la poblacional nacional se identifica fuera de 
la heteronormatividad, de ellos el 30,1\% declaró haber sufrido discriminación en los últimos 12 meses por su orientación sexual. Sin embargo, es probable que la cifra sea aún más alta, ya que muchas personas podrían no haber compartido su orientación sexual abiertamente.

La discriminación hacia las personas LGBTI+ en forma de violencia, agresión, rechazo, maltrato, segregación y cualquier otra privación de los derechos básicos de un individuo (Boivin, 2014), atenta contra su desarrollo personal, familiar, académico, profesional e incluso contra su salud. En la ENADIS además se reporta que el 64,4\% de los encuestados considera que en poco o nada se justifica que dos personas del mismo sexo vivan como pareja, siendo el estado de Nuevo León uno de los más altos con un $72,2 \%$. A pesar de que el $66 \%$ de las personas encuestadas considera que se respetan poco o nada los derechos de los homosexuales, un $32 \%$ del total no estaría dispuesta a rentar una habitación a personas homosexuales y 4 de cada 10 personas no estarían de acuerdo con que una persona gay o lesbiana fuera electa para la Presidencia de la República (Consejo Nacional para Prevenir la Discriminación [CONAPRED], 2018).

Adicionalmente, el 75\% de los hombres homosexuales y el 50\% de las mujeres homosexuales han sufrido algún tipo de discriminación dentro de los salones de clases (Gómez, 2015), en tanto que en el informe de la Primera Encuesta sobre Homofobia y el Mundo Laboral en México se reportó que al 14\% de los encuestados se les negó una oportunidad laboral y su identidad sexual y/o de género fue motivo de despido para el 10\% (Vela, 2017).

La homofobia sigue presente en el mundo. Existen 72 naciones que criminalizan las relaciones de las personas del mismo sexo, 33 en África, 23 en Asia, 10 en América y 6 en Oceanía, en 13 de las cuales se penalizan con la muerte. Datos de la Asociación Internacional de Lesbianas, Gays, Bisexuales, Trans e Intersex (ILGA, por sus siglas en inglés) indican que México es uno de los 124 países donde no se ejerce ninguna ley en contra de las relaciones homosexuales y junto con 8 naciones más en el mundo prohíbe la discriminación basada en la preferencia sexual (Carroll y Mendos, 2017), lo que podría representar un descenso de la discriminación por orientación sexual en México.

En 2003 se aprobó la Ley Federal para Prevenir y Eliminar la Discriminación, a partir de entonces los 32 estados han emitido leyes sobre discriminación. El matrimonio entre personas del mismo sexo entró en efecto el 4 de marzo del 2010 en Ciudad de México otorgando igualdad jurídica a todas las personas sin importar su orientación sexual y, a la fecha, 11 Estados reconocen el matrimonio igualitario. En 2011 se modificó el Artículo 1 de la Constitución quedando prohibida la discriminación por preferencias sexuales de un individuo que incluyen la orientación sexual y la identidad sexo-genérica (CONAPRED, 2018). Asimismo, la Secretaría de Salud (2017) elaboró un protocolo de atención para personas LGBTI+ garantizando el acceso sin discriminación a los servicios de salud. 
No obstante, prevalece una sociedad heteronormativa en la que las características de género son la "configuración predeterminada" de todos los seres humanos y la orientación sexual va ligada directamente con el sexo determinado al nacer. Esto afecta directamente a las personas con una orientación sexual no heteronormada, ya que quedan fuera de esta estructura establecida por la serie de creencias socioculturales que se ven reflejadas en el núcleo familiar que las mantiene invisibles (Hébert et al., 2017).

\section{Estereotipos, prejuicios y homofobia}

La sexualidad se expresa socialmente de diferentes maneras (Benavente y Vergara, 2006; Organización Mundial de la Salud [OMS], 2018), no siempre de la manera en que la sociedad lo marca. Al nacer, lo único que está definido es el sexo anatómico. Zupančič (2017) postula la importancia de saber qué es el sexo. El ser humano no nace sexuado y la anatomía es insuficiente para la definición sexual (Elfakir, 2013), así que mujer u hombre devienen como persona sexuada a partir de su desarrollo psíquico, de sus relaciones intersubjetivas y de la cultura (Flax, 1995), construyéndose y asumiéndose (Butler, 2002), dando paso a su identidad de género, su orientación sexual y su rol sociosexual (Shively y De Cecco, 1977). La identidad sexual supone el reconocimiento y la aceptación sexual esencialista-anatómica (Mosher, 2001; Fausto-Sterling, 2006) o socioconstruida, fluida y múltiple a través de la interacción con otros (Brubaker y Cooper, 2000), un sexo socializado (Laqueur, 1994).

La orientación se refleja en la atracción hacia otro en el plano emotivo, romántico, afectivo o sexual (Atienza, 2015), denominada como heterosexual (hacia el sexo opuesto al propio), homosexual (hacia el mismo sexo), bisexual (atracción hacia ambos sexos; American Psychological Association [APA], 2008), o asexual (ausencia de atracción; Planned Parenthood, 2018), en un continuo que va de la exclusividad heterosexual hasta la exclusividad homosexual quedando fuera la asexualidad (Corona y Funes, 2015).

Los estereotipos de género son ideas plasmadas y transmitidas desde que nace un individuo mediante un proceso social (Colás y Villaciervos, 2007) e implican todos los papeles, funciones y expectativas adjudicados a hombres y mujeres (Melero, 2010; Rocha y Díaz-Loving, 2011). Desde la niñez se fomentan las nociones de que el hombre tiene que ser fuerte, viril y proveer el sustento a la familia y que la mujer es más vulnerable y se hace cargo de la casa (Inda, 1996). Cualquier desviación de lo socialmente establecido es desaprobada, rechazada y criticada y vista como algo diferente e incomprendido (Ortiz-Hernández, 2005; Saeteros et al., 2014; Mosteiro y Porto, 2017) y permite la perpetuación del estigma social por ser un individuo con defectos de carácter por falta de voluntad (Goffman, 2006) que luego pasa a la acción 
al discriminarlo y privarlo injustamente de sus derechos y oportunidades (United Nations Educational, Scientific and Cultural Organization [UNESCO], 2016). En México, entre 66,9\% y 83,6\% de personas LGBTI+ sufren de discriminación (Lozano y Salinas, 2016; Mendoza et al., 2016).

El problema comienza en la familia al estigmatizar la diversidad sexual e imponer la adaptación de los hijos a la heteronormatividad dominante, eliminando todo lo incompatible (Boivin, 2014). A este respecto, 4 de cada 10 personas de la comunidad LGBTI+ reconocieron vivir en un entorno violento que limitaba su desarrollo personal y social (CONAPRED, 2018) e informaron que era necesario salir de su entorno próximo para ser aceptados o decidían permanecer y prolongar la "salida del clóset" por temor al rechazo por parte sus seres queridos (Corral, 2018).

Las actitudes y comportamientos que performan las relaciones sociales denigrando las condiciones sexuales no heteronormadas da lugar al heterosexismo, postura ideológica que busca negar, denigrar y estigmatizar cualquier comportamiento, relación o comunidad no heterosexual (Herek, 2004; Hunter, 2007). Pensar que la heterosexualidad es lo normal y lo aceptable lleva a las personas a comprometer la tolerancia social e invisibilizar a las identidades LGBTI+ (Hébert et al., 2017), al devaluarlas y desacreditarlas y connotarlas como desviadas y amenazantes (Piedra et al., 2013). Adicionalmente, puede dar lugar a homolesbotransfobia, maltrato explícito sutil como el silencio y la exclusión (Cavallero et al., 2013) y a dar por sentadas las "verdades" heterosexuales alegando la supuesta necesidad humana de reproducirse (Warner, 1993; Wittig, 2006).

Además, el heterosexismo se asocia a problemas de salud mental graves con indicadores de morbilidad psiquiátrica en individuos que se identifican como parte de la comunidad LGBTI+ (Simpson et al., 2016), dado que sus características indeseadas merecen ser erradicadas (Hunter, 2007), lo que apuntala igualmente el tránsito a la homofobia, expresión individual de ideologías socialmente institucionalizadas (Herek, 2000, 2004).

Otras formas de discriminación comunes son la plumofobia y el contagio del estigma. La primera se puede manifestar aún y cuando la persona homosexual sea aceptado en su orientación sexual, pero no en la transgresión de los roles de género: varones afeminados y mujeres amasculinadas; en tanto que la segunda refiere a la exclusión de quienes aceptan y se alían con la persona homosexual y se arriesgan a ser injuriados o desacreditados (Pichardo, 2012).

Actualmente, se está creando más conciencia social hacia temas de homosexualidad y orientación sexual, atracción o deseo afectivo-erótico que se van descubriendo al inicio de la adolescencia. No obstante, la homofobia basada en estereotipos, prejuicios y estigmas se continúa expresando en actitudes y conductas discriminatorias que ponen en riesgo la igualdad, la dignidad, los derechos y las libertades de toda persona y podría llegar a generar diversos tipos de violencia 
(Human Rights Watch, 2014; CONAPRED, 2018; Smith, 2018), en acto u omisiones que pueden ocasionar daño o sufrimiento físico, psicológico o sexual, llegando incluso al homicidio (Instituto Nacional de las Mujeres [INMUJERES], 2009). De acuerdo con la Comisión Ciudadana de Crímenes de Odio por Homofobia, en México se reportaron 1.310 asesinatos por odio homofóbico entre 1995 y 2015 (Vera, 2018), 81 de ellos en Nuevo León y 3 de cada 10 contra jóvenes de entre 18 y 29 años (Cubero, 2016).

\section{Proceso de reconocimiento de la orientación sexual homosexual e inclusión familiar}

Al nacer, lo único definido en el ser humano es el sexo anatómico que evoluciona para definirse como mujer u hombre, a partir de su desarrollo biopsíquico en la infancia y de las interacciones con los demás dentro de un marco cultural que les da significado como femenino y masculino según características, expectativas y espacios físicos y simbólicos (Osborne y Molina, 2008). Cada individuo asume el género como real cuando lo social se convierte en autorrepresentación (De Lauretis, 2015). La homosexualidad es una categoría socialmente reconocida de una persona al asumir su manera de pensar, sentir y actuar al declarar su identidad sexual como gay/lesbiana (Savin-Williams y Diamond, 2000). Al instaurar y mantener un género inteligible con coherencia y continuidad entre sexo, género, práctica sexual y deseo, el individuo se puede construir y asumir más allá del marco binario impuesto por la sociedad (Butler, 2002,2007) para después decidir cuándo, cómo y ante quién revelar su identidad sexual (Lingiardi et al., 2015).

La orientación sexual refiere al patrón de atracción sexual-erótico o amoroso hacia un determinado grupo de personas definidas por su género o su sexo y es independiente de la identidad de género. Aunque la atracción hacia el sexo opuesto pueda tener un origen temprano, llegar a reconocer la identidad sexual es un proceso (Klein et al., 2015; Goldberg, 2016; Forsell, 2017). Cuando los adolescentes empiezan a descubrir su orientación sexual no heteronormada, lo comentan con pocas personas debido al fuerte impacto emocional, prefiriendo evadirlo y vivirlo de una manera solitaria por miedo a desilusionar a sus seres queridos (Quintana y Vásquez, 2003; Flores y Solís, 2017) o a no ser aceptados y ser discriminados (Mitchell, 2012). A medida que crecen es más probable que vayan aceptando su orientación homosexual sin tanto temor como lo sentían cuando eran más jóvenes (Marshal et al., 2011). Asimismo, en las familias donde existe una educación sobre sexualidad existen menos prejuicios, estigmas y discriminación hacia los hijos al expresar su orientación sexual y a la aceptación de sus parejas (White et al., 2016).

Cuando llega a aceptarse a sí mismo y empieza el proceso de "salir del clóset" - en lugar de mantenerse oculto-, cualquiera sea la forma en la que los padres de 
familia lleguen a conocer sobre la orientación sexual de su hijo, la mayoría de las veces viene acompañada de sentimientos negativos y desorganización de emociones (López, 2006; Maroto, 2006; Ryan, 2009). Sin embargo, después de la reacción inicial a esa nueva realidad, suele mejorar la vida emocional y social de los hijos y las relaciones dentro de la familia la mayoría de las veces (López, 2006).

Impacto de revelar la identidad sexual ante la familia. Las personas tienen la necesidad de ser autónomas, de poder autodeterminarse y ser ellas mismas y actuar de acuerdo con sus valores y sentimientos (Deci y Ryan, 2000; Ryan y Deci, 2000). Autodeterminarse como homosexual puede ser validado por los demás (Ryan et al., 2015) o frustrado por un amor contingente al cumplimiento de expectativas específicas que sacrifica la autonomía para preservar la relación (Assor et al., 2004).

$\mathrm{La}$ angustia psicológica de las personas de la comunidad LGBTI+ tiene una incidencia significativamente más alta en países sin leyes o políticas que protejan a las minorías sexuales contra la discriminación y la violencia. Estresores distales emergentes de los procesos estructurales culturales - p.e., discriminación y victimización-, y proximales surgidos de la construcción personal identitaria (Meyer, 1995), pueden derivar en temor a la reacción ante la revelación de la identidad sexual a los demás. La familia puede ser una fuente adicional de incomodidad y preocupación (Paul et al., 2002; Frost y Meyer, 2009; Barrientos y Cárdenas, 2013; Lingiardi et al., 2015).

El miedo de perder a su familia y amigos, ver su trabajo afectado o hasta llegar a perderlo; el temor a la manera hostil en que la sociedad se dirige al tema de la homosexualidad, y a la falta de oportunidades que puede esto traer, lleva a que la mayoría de los homosexuales prefieran vivir en silencio, sin poder expresar su identidad sexual libremente, sin poder tener una vida amorosa pública como las personas con una orientación heterosexual, obligándolos a llevar una doble vida, aparentando por temor a decepcionar a la familia por no poder cumplir con sus expectativas, al no ser las personas que ellos esperaban (Flores y Solís, 2017), con todas las consecuencias que la presión de esta simulación llegar a implicar (Hinojosa y Betancourt, 2007).

$\mathrm{Al}$ pertenecer a un grupo estigmatizado, las personas homosexuales en ocasiones asumen la evaluación negativa que la sociedad hace de ellas, conflictúandose consigo mismas en lo que se ha denominado la homofobia internalizada, que refleja la relación entre la persona y su ambiente (Frost y Meyer, 2009) al colocarse en la posición de inferioridad que la sociedad les dicta (Herek, 2004; Castañeda, 2012), estigma que "borra o anula el resto de elementos de su identidad" (Pichardo, 2012, p. 111).

La homofobia internalizada fortalece la aparición de sentimientos negativos hacia la identidad sexual o de género propia (Ortiz-Hernández, 2005; Baute, 2018), despertando una sensación de culpa, vergüenza y vulnerabilidad enraizada en la discriminación, violencia y estigma que experiencian (Meyer, 1995, 2003; OrtizHernández, 2005; Herek y Garnets, 2007; Frommer, 2018). También, se asocia a 
insatisfacción e inestabilidad en sus relaciones de pareja (Lewis et al., 2014; Szymanski et al., 2016; Li y Samp, 2019).

Hatzenbuehler (2009) extendió la teoría del estrés de las minorías con relación a los estresores distales que impactan la salud mental y propuso mecanismos generales (p.e., aislamiento social) y grupales (p.e., homonegatividad internalizada). Resultado de la discriminación se ha observado la aparición o complicación de psicopatologías (Jackson y Mohr, 2016); entre ellas, síntomas internalizados como ansiedad (Lehavot y Simoni, 2011), desregulación emocional (Hatzenbuehler et al., 2009), depresión (Berghe et al., 2010; Lehavot y Simoni, 2011) y la presencia de ideas suicidas que expresan la intensificación o la cronicidad de los procesos depresivos (Cochran y Mays, 2006; Russell,2006; Pineda, 2018). También, se asocia de manera parcial con estigma internalizado (Calton et al., 2016), expectativas de rechazo (Feinstein et al., 2012), rumiación (Hatzenbuehler, 2009), aislamiento social (Hatzenbuehler et al., 2009), abuso de sustancias y conductas sexuales de riesgo (Andújar et al., 2006; Greene et al., 2006).

En estudios en México se ha encontrado la homosexualidad internalizada asociada con depresión (Lozano et al., 2017), intentos e ideación suicida (GranadosCosme y Delgado-Sánchez, 2008) y con abuso de alcohol (Baruch, 2015; Mendoza et al., 2016; Lozano et al., 2017).

Ante todas estas barreras sociales y subjetivas, la decisión de cuándo y cómo revelar su identidad sexual supone trascender los condicionamientos culturales, las normas del sistema sexo-género-orientación (Pichardo, 2012), el temor a ser rechazado y aislado socialmente (Borrero-Bracero, 2008), el afrontamiento de la homofobia internalizada (Russell y Richards, 2003). Supone, entre otros elementos, que la persona se haya construido y asumido con un género inteligible (Butler, 2002, 2007); la aceptación y el apoyo en sus relaciones parentales e interpersonales con sus pares (Russell y Richards, 2003) que le brinden visibilidad y estabilidad (Fernández y Vázquez, 2013); al tiempo que fortalecen su resiliencia (Gil, 2007; Hernández, 2010) y, con ella, la voluntad para dejar de lado las circunstancias históricas actuales que mantienen las desigualdades de valor y poder entre personas y colectivos sociales (Stolcke, 2004).

Por otro lado, al declararse como homosexual el hijo, la familia puede entrar en una etapa de crisis para alcanzar luego una nueva homeostasis; pero existen ocasiones en las que la dinámica familiar se puede rigidizar y llevar a una actitud de rechazo (Lingiardi et al., 2015). Los padres de familia suelen dar por hecho la identidad de sus hijos conforme a la heteronormatividad (Cedillo, 2017), por lo que con la revelación pueden temer por lo que sus amistades, miembros de la sociedad y demás personas puedan pensar sobre la sexualidad de sus hijos y sus habilidades parentales, prefiriendo no hablar de ello con las personas fuera de su núcleo primario. Este tipo de situación es muy riesgosa, especialmente para los adolescentes que pueden experimentar una 
profunda sensación de aislamiento, capaz de interferir en su desarrollo y aumentar el riesgo de depresión, abandono escolar y del hogar prematuramente, así como conductas autodestructivas (p.e., abuso de sustancias y suicidio; Baiocco et al., 2015; Goldberg, 2016). Es así como los síntomas de depresión se han asociado a estilos de afrontamiento evitativos - lo que puede demorar aún más el momento de develar su identidad ante los padres - y a escaso apoyo social. Incluso, enfrentan altos niveles de estrés y sentimientos de culpabilidad (Varas-Díaz et al., 2004; De Bedout, 2008), autolesiones sin riesgo suicida (Buresová, 2016) e intento suicida (Posner et al., 2014; Hermosillo-De la Torre et al., 2015).

De ahí que quien va a develar su identidad de género prefiera hacerlo en contextos en los que las personas apoyen la autonomía y la auténtica autoexpresión, ya sea la propia familia (Legate et al., 2012; Ryan et al., 2015) o los amigos u otras personas estigmatizadas igual que ellos y con quienes normalmente se sienten más seguros y con las que tienen más apertura en cuanto a sus temas de conversación, para ser ellos mismos (Lingiardi et al., 2015; Goldberg, 2016) y además recibir su apoyo incondicional (Kwon, 2013; Belous et al., 2015; Verrelli et al., 2019). El apoyo social y la visibilización favorecen el proceso de aceptación y desarrollo de la identidad positiva como persona homosexual, una identidad autorreferenciada, que es en sí misma un proceso de resiliencia (Gil, 2007; Hernández, 2010). Esto es, para desarrollar la resiliencia el joven activa y utiliza sus fortalezas y recursos al transitar la adversidad desde el peor de los temores - ser expulsado fuera del flujo de la interacción humana (Csikszentmihalyi, 1997) - , resistiendo al rechazo, manteniendo un buen nivel de autoestima (Borrero-Bracero, 2008) y confrontando la homofobia internalizada (Rusell y Richards, 2003).

Por último, aunque ya se ha reportado la relación directa existente entre la aceptación de la homosexualidad personal y familiar y la depresión (GranadosCosme y Delgado-Sánchez, 2008; Legate et al., 2012; Ryan et al., 2015; Pineda, 2018) y si bien hay trabajos que describen la postergación de la revelación de la homosexualidad en la adolescencia (Quintana y Vásquez, 2003; Marshal et al., 2011; Flores y Solís, 2017), son pocos los trabajos en torno a establecer el efecto mediador de la edad; es decir, esta tercera variable como explicativa de la relación entre la depresión y la aceptación de la homosexualidad. Por tanto, en el presente estudio se buscó demostrar de qué manera se explica la depresión comúnmente asociada a la falta de aceptación personal y familiar de las personas homosexuales. Para lograrlo, se probó el efecto mediador que la edad $(M)$ tiene sobre el efecto directo de la falta de aceptación personal y familiar $(X)$, y como predictor focal de la depresión $(Y)$, la variable resultado. 


\section{Metodología}

La presente investigación es un estudio cuantitativo, explicativo, de corte transversal. Se convocó a hombres y mujeres homosexuales mayores de edad a participar en forma voluntaria y anónima. Se logró reclutar a 354 participantes de entre 18 y 56 años $(M=27.95, D T=7.33)$.

Un análisis de sensibilidad estadística mostró que con este tamaño muestral al comparar dos grupos de interés - $187(52,8 \%)$ hombres homosexuales y $159(44,9 \%)$ mujeres homosexuales, excluyendo a $8(2,3 \%)$ que se identificaron como otro-, con un nivel alfa de .05 , se puede detectar un tamaño del efecto tan pequeño como $d=.30$ si se considera una potencia de .80 , o de $d=.39$ si se considera una potencia de .95 (Faul et al., 2007).

Los instrumentos utilizados fueron:

Depression Anxiety Stress Scale (DASS-21), adaptación para población mexicana. Autoinforme de 3 subescalas con 7 ítems cada una que miden depresión general, sobreexcitabilidad y tensión (Lovibond y Lovibond, 1995). Para este estudio solamente se utilizó la subescala de depresión general que mide el estado de ánimo disfórico; es decir, la baja afectividad negativa (p.e., "Me sentí triste y deprimido"), con cuatro opciones de respuesta que van del 3 (se aplica mucho a mí la mayor parte del tiempo) al 0 (no se aplica nada a mí). Se consideran puntajes normales (0-9), bajos (10-13), moderados (14-20), severos (21-27) y extremadamente severos (28+). Tuvo una consistencia interna satisfactoria $(\alpha=.940)$.

Cuestionario de Aceptación Personal y Apoyo Familiar de 12 reactivos que mide niveles de apoyo familiar, depresión, autoestima y discriminación (p.e., ¿Piensa usted que si fuera heterosexual tuviera una mejor relación con sus padres?; Cano, 2013). En una escala Likert de cinco puntos, 1 = nunca, 5 = siempre; su confiabilidad para el estudio fue aceptable $(\alpha=.672)$.

Cuestionario sociodemográfico diseñado para el estudio solicitando información sobre género, edad, si contaban o no con pareja, si tenían o no familiares con orientación homosexual, si su pareja era aceptada dentro de su familia.

La aplicación de los instrumentos de medición se realizó a través de la plataforma Google Forms, donde se contestaron los instrumentos. Los participantes fueron reclutados acudiendo a distintos colectivos de personas homosexuales del área metropolitana de la ciudad de Monterrey los meses de agosto a octubre de 2018. El consentimiento informado incluía información sobre el propósito del estudio, e información de contacto de las investigadoras. Toda la información fue dada de manera anónima y confidencial y sin remuneración económica ni de ninguna especie.

El plan de análisis estadístico consistió en examinar inicialmente las propiedades psicométricas de los instrumentos utilizados. Posteriormente, se realizaron cálculos de estadística descriptiva como media, desviación estándar para luego realizar un 
análisis de comparación de medias para muestras independientes — hombres y mujeres homosexuales - con las variables de interés.

Antes de llevar a cabo el análisis de mediación, se realizó un análisis de correlación bivariada de Pearson para evaluar la asociación de la depresión con la aceptación personal y el apoyo familiar para evaluar la intensidad y la linealidad de la relación. Para el análisis de mediación, se utilizó una regresión lineal múltiple a partir de los datos recopilados para examinar la interacción de las variables utilizando la herramienta computacional para el programa estadístico para las ciencias sociales (SPSS, por sus siglas en inglés) de libre acceso macro PROCESS (model 4, bootstrapping 10,000 samples, 95\% CI; Hayes, 2018).

\section{Resultados}

\section{Participantes}

La edad media de las 354 personas que contestaron los cuestionarios fue de $28,42 \%, D T=7.08 ; 170(50,57 \%)$ reportaron tener pareja y $175(49,43 \%)$ no. Asimismo, más de la mitad de la muestra $(199,56,21 \%)$ reportó tener un familiar homosexual: 41 (11,58\%) un hermano/a, 108 (30,51\%) un primo/a, y $50(14,12 \%)$ un tío/a.

Poco menos de la mitad indicó poder hablar abiertamente de su homosexualidad. Cuando se analizó la variable de edad y la respuesta a esta pregunta con una prueba $t$ de Student para muestras independientes, se encontró una diferencia estadísticamente significativa que indica que las 168 personas que contestaron Sí, implicando una mayor aceptación de la propia homosexualidad, tienen una edad mayor que las 199 personas que contestaron $\mathrm{No}\left(M_{S I}=29.65, D T=7.36 ; M_{N O}=26.52, D T=7.01 ; t_{(365)}=\right.$ $-4.160, p<.001)$.

\section{Modelo de mediación}

Previo a realizar el análisis de mediación, se estableció la correlación entre depresión y aceptación personal y familiar, encontrando una relación negativa de nivel bajo a moderado estadísticamente significativa $(r=-.448, p=.001)$.

Posteriormente, se realizó un análisis de regresión múltiple para examinar la interacción de las variables utilizando la herramienta computacional para el programa estadístico para las ciencias sociales (SPSS, por sus siglas en inglés) de libre acceso macro PROCESS. Para este análisis en específico se consideró la Aceptación Personal y Familiar como la variable predictora $(X)$, con la Depresión como la variable de resultado $(Y)$, en tanto que la Edad $(M)$ fue la variable mediadora. 
$\mathrm{E}$ modelo mediacional que mostró un buen ajuste $\left(R^{2}=.26\right)$ indica que una menor aceptación personal y familiar de la homosexualidad tiene un efecto directo sobre la depresión ( $\beta=-0.05, C E=.006$, IC 95\% [-.065, -0.04], incrementándola $\left(F_{(349)}=64.49, p<.001\right)$. Este efecto tiende a desaparecer el impacto negativo del rechazo parental en el estado de ánimo del hijo homosexual entre más edad tenga (Figura 1).

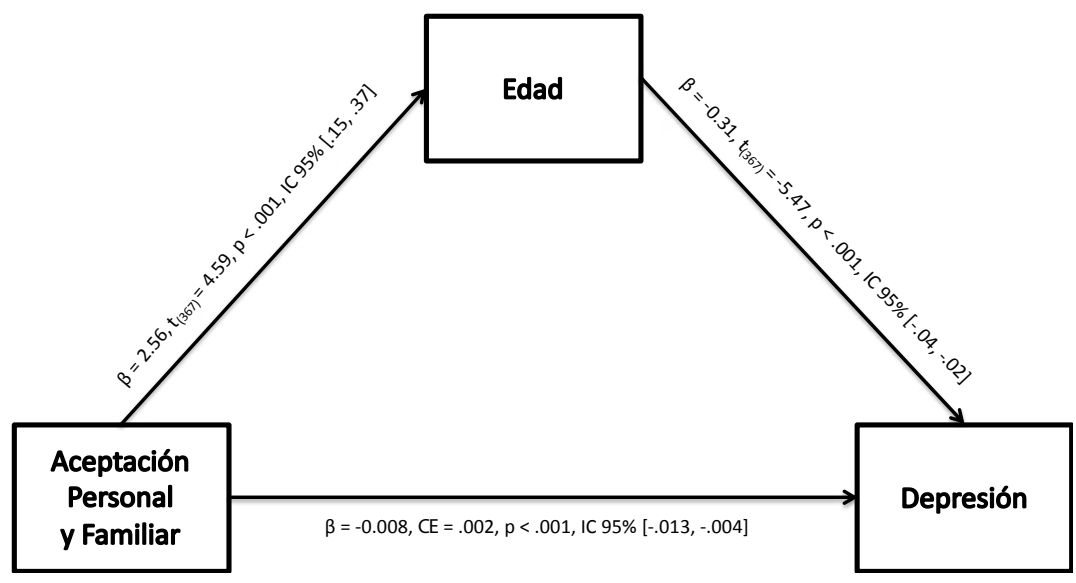

Figura 1. Modelo mediacional explicativo del impacto de la aceptación personal y familiar sobre la depresión que se explica a través de la edad.

Fuente: elaboración propia.

\section{Discusión}

El objetivo de la presente investigación era demostrar de qué manera se explicaba la depresión comúnmente asociada a la falta de aceptación personal y familiar de las personas homosexuales. Se encontró que la edad de la persona homosexual explica el nivel de depresión que puede llegar a desarrollar como consecuencia de la desaprobación parental. Cuando la persona homosexual es mayor, hay una mayor aceptación de sí misma y de parte de su familia, lo que disminuye los niveles de depresión independientemente del rechazo de los padres, en línea con lo que proponen Marshal et al. (2011). También, indica que al pasar el tiempo los individuos se sienten más aceptados por sus padres (López, 2006), quienes han ido transitando del suspenso de empezar a pensar en formas de vida cotidiana y arreglos íntimos, al conflicto moral que implica cualquier arreglo sexoafectivo y parental, hasta considerar nuevas y diversas fórmulas del anterior modelo familiar hetero-biparental (Vespucci, 2017). 
Este hallazgo del rol mediador de la edad es particularmente relevante ya que permite una nueva visión de la interacción entre la tríada aceptación personal y familiar-depresión-edad. De acuerdo con datos de la Encuesta Nacional de los Hogares -ENH- (INEGI, 2018b), en México la condición de sentimientos de depresión se incrementa con la edad, pasando de 14,6\% de 7 a 14 años, a 25,8\% de 15 a 29 años, a 34,3\% de 30 a 49 años. El dato que el efecto de la desaprobación personal y parental sobre la depresión tiende a desaparecer con la edad es contrario a la prevalencia de la depresión en México. Esto podría explicarse desde distintas perspectivas. De acuerdo con Gil (2007) y Hernández (2010) podría ser resultado del desarrollo de la resiliencia del joven en su proceso personal de aceptación y desarrollo de su identidad como homosexual. Asimismo, el apoyo social y la visibilización tempranos ya sea en casa, con pares o en colectivos, favorece su motivación y el uso de sus fortalezas para afrontar su situación de vida, sintiéndose orgulloso de quién es (Mohr y Fassinger, 2003; Richardson, 2004; Balsam y Mohr, 2007; Kwon, 2013).

Es clara entonces la importancia de la inclusión del hijo en la familia aceptándolo como antes de conocer su orientación sexual (Ryan, 2009), ya que el rechazo y el sufrimiento de sus familiares son motivo de tristeza en las personas homosexuales (Granados-Cosme y Delgado-Sánchez, 2008; Ryan et al., 2009) y la aceptación ayuda a recuperar la autoconfianza, seguridad y autonomía (Ryan et al., 2015; Goldberg, 2016; Zambrano et al., 2017).

Asimismo, el modelo explicativo coincide con lo propuesto por Marshal et al. (2011) en relación a que a medida que los jóvenes van creciendo es más probable que vayan aceptando su homosexualidad sin tanto temor, como lo sentían cuando eran más jóvenes. Esto podría relacionarse con el tiempo que invierten en decidir cuándo, cómo y a quién develar su homosexualidad (Legate et al., 2012) ante la posible culpa (Frommer, 2018) y la expectativa de riesgo de desilusionar (Castañeda, 2012; Mitchell, 2012) o incumplir las expectativas de sus padres (Flores y Solís, 2017), inconformar a su familia (Heatherington y Lavner, 2008; Lingiardi et al., 2015) o enfrentar un posible rechazo o aversión social (Castañeda, 2012) por no cumplir con lo que la sociedad demanda (Butler, 2017).

Adicionalmente y de acuerdo con White et al. (2016), podría estar asociado a que en las familias exista mayor inclusividad — más de mitad de la muestra (199, $56,21 \%)$ reportó tener un familiar homosexual- y, por tanto, se observan menos prejuicios, estigmas y discriminación hacia los hijos al expresar su orientación sexual; o bien, a que al ser mayor de edad por lo general se es económicamente más estable e independiente, lo que crea mayor seguridad para que el hijo acepte y comunique su orientación sexual.

No obstante, Lipkin (2018) reporta que los jóvenes actualmente están pasando por su proceso de salir del clóset a una edad más temprana, que pudiera asociarse con una comunicación familiar abierta y auténtica como lo que refieren Lingiardi et al. 
(2015), o bien contar con una familia "elegida", es decir, personas que les brinden comodidad y confianza para hablar sobre temas de amor y sexualidad, sintiéndose seguros al develar su identidad (Malley y Tasker, 2007).

Haber identificado la interacción de las variables en el modelo explicativo resalta la importancia que como sociedad tenemos ante la comunidad LGBTI+. Una mayor tolerancia, entendimiento y aceptación por la diversidad sexual y familias más educadas en el amor y el respeto por las elecciones de sus hijos, favorecerían el proceso de develar la identidad no heteronormada. A decir de Meler (2016), podrían recuperar su voz y espacio en el mundo, visibilizándolos sin cuestionamientos ni etiquetas y para reducir los riesgos asociados a la depresión, efecto directo de la falta de aceptación personal y familiar.

Limitaciones de esta investigación son las propias de los autoinformes como la tendencia a magnificar o minimizar las respuestas y la deseabilidad social, tendencia a responder creando una imagen más favorable de sí mismo. Del mismo modo, la información que se cuenta sobre la temática en México es incipiente y además no se incluyeron en la muestra personas del resto de la comunidad LGBTI+, por lo cual se recomienda la participación representativa de cada segmento de esta comunidad en futuras líneas de trabajo, incluyendo incluso a sus minorías - p.e., asexuales, intersexual, entre otros-, así como ampliar los rangos de edad.

\section{Conclusiones}

Es importante educar sobre el tema de diversidad sexual para minimizar el impacto negativo en el proceso de aceptación. Aparentemente, la fuerza del heterosexismo sigue forzando a las personas homosexuales a la invisibilidad ya que, como se corroboró en el estudio, más de la mitad de los participantes declaran su homosexualidad al garantizar el anonimato de sus respuestas, sin embargo, reportan no poder hablarlo abiertamente. Esta problemática específica amerita reconocimiento, atención y respeto de manera de poder reducir la fisura entre los derechos de las personas homosexuales y la rígida regulación social del comportamiento.

Asimismo, se requiere implementar políticas públicas que garanticen la reparación de la injusticia social de que son objeto no solo las personas homosexuales, sino también todas aquellas que forman parte de las comunidades LGTBI+, de manera que se integren de manera abierta y activa al desarrollo social y económico del país.

Por último, queda pendiente seguir estudiando si develar la identidad sexual se explica por la fuerza de asociación con factores sociocontextuales (p.e., apoyo social, visibilización) o por recursos personales (p.e., resiliencia, ajuste psicológico). 
El rol de la edad en el estado de ánimo asociado al rechazo parental de la homosexualidad

\section{Referencias}

American Psychological Association (APA). (2008). Answers to your questions: For a better understanding of sexual orientation and homosexuality. https://www.apa.org/topics/lgbt/ orientation.pdf

Andújar, I., Nieves, L., Toro, J. y Varas, N. (2006). Strengths and vulnerabilities of a sample of gay and bisexual male adolescents in Puerto Rico. Revista Interamericana de Psicología, 40(1), 59-68.

Assor, A., Roth, G. y Deci, E. L. (2004). The emotional costs of parents' conditional regard: A self-determination theory analysis. Journal of Personality, 72, 47-88. doi: 10.1111/j.00223506.2004.00256.x

Atienza, C. M. (2015). Diversidad de género, minorías sexuales y teorías feministas: Superposiciones entre las teorías de lesbianas, gays, bisexuales y transexuales y el feminismo en la reformulación de conceptos y estrategias político-jurídicas. Dykinson.

Baiocco, R., Fontanesi, L., Santamaria, F., Ioverno, S., Marasco, B., Baumgartner, E., ... Laghi, F. (2015). Negative parental responses to coming out and family functioning in a sample of lesbian and gay young adults. Journal of Child and Family Studies, 24(5), 1490-1500. doi: 10.1007/s10826-0149954-z

Balsam, K. y Mohr, J. J. (2007). Adaptation to sexual orientation stigma: A comparison of bisexual and lesbian/gay adults. Journal of Counseling Psychology, 54(3), 306-319. doi: 10.1037/00220167.54.3.306

Barrientos, J. y Cárdenas, M. (2013). Homofobia y calidad de vida de gay y lesbianas: Una mirada psicosocial. Psykhe (Santiago), 22(1), 3-14. https://dx.doi.org/10.7764/psykhe.22.1.553

Baruch, R. (2015). Reporte de resultados de la Encuesta sobre Uso de Drogas en Población LGBT en México. Espolea, A. C.

Baute, M. (2018). Conceptos. Lulu.

Belous, C. K., Wampler, R. S. y Warmels-Herring, T. (2015). Gay male adaptation in the coming-out process. Journal of Gay y Lesbian Mental Health, 19(1), 55-71. https://dx.doi.org/10.1080/193597 05.2014 .921265

Benavente, M. C. y Vergara, B. M. (2006). Sexualidad en hombres y mujeres: diversidad de miradas. Flasco.

Berghe, W. V., Dewaele, A., Cox, N. y Vincke, J. (2010). Minority-specific determinants of mental well-being among lesbian, gay, and bisexual youth. Journal of Applied Social Psychology, 40(1), 153166. https://doi.org/10.1111/j.1559-1816.2009.00567.x

Boivin, R. R. (2014). "Se podrían evitar muchas muertas”. Discriminación, estigma y violencia contra minorías sexuales en México. Sexualidad, Salud y Sociedad - Revista Latinoamericana, 16, 86-120. http://dx.doi.org/10.1590/S1984-64872014000100006

Borrero-Bracero, N. (2008). De la vulnerabilidad a la resiliencia: las investigaciones sobre la "salida del closet" de jóvenes gay puertorriqueños. Ciencias de la Conducta, 23(1), 25-54.

Brubaker, R. y Cooper, F. (2000). Beyond identity. Theory and Society, 29, 1-47. doi: 10.1023/A:1007068714468

Buresová, I. (2016). Self-harm in adolescence. Peter Lang Academic Research. 
Butler, J. (2002). Cuerpos que importan. Sobre los limites materiales y discursivos del "sexo". Paidós.

Butler, J. (2007). El género en disputa. Paidós.

Butler, J. (2017). Deshacer el género. Paidós.

Calton, J., Bennett, L.y Gebhard, K. (2016). Barriers to help seeking for lesbian, gay, bisexual, transgender, and queer survivors of intimate partner violence. Trauma, Violence y Abuse, 17, 585-600. https://doi. org/10.1177/1524838015585318

Cano, J. E. (2013). La influencia de la aceptación familiar en personas homosexuales, caso atendido en la clínica de la Universidad José Matías Delgado (tesis de grado). Universidad Dr. José Matías Delgado, San Salvador.

Carroll, A. y Mendos, L. R. (2017, mayo). Homofobia de Estado 2017. Estudio jurídico mundial sobre la orientación sexual en el derecho: criminalización, protección y reconocimiento. Asociación Internacional de Lesbianas, Gays, Bisexuales, Trans e Intersex (ILGA).

Castañeda, M. (2012). La experiencia homosexual: para comprender la homosexualidad desde dentro y desde fuera. Paidós.

Cavallero, L., Mines, A. y Volpin, L. (2013). Cómo opera el heterosexismo en el sistema de salud actual en el Área Metropolitana de Buenos Aires (AMBA): Una aproximación a partir de experiencias de trans femeninas, trans masculinos, lesbianas y gays. Anuario de Investigaciones, 4, 1-17.

Cedillo, C. M. (2017). Ideación suicida en hombres gay y bisexuales jóvenes. Revista Electrónica Sobre Cuerpos Académicos y Grupos de Investigación, 4(8). http://www.cagi.org.mx/index.php/CAGI/ article/view/142/255

Csikszentmihalyi, M. (1997). Fluir: una psicología de la felicidad. Editorial Kairós.

Cochran, S. D. y Mays, V. M. (2006) Prevalencia de trastornos mentales y abuso de sustancias entre lesbianas y gays. En A. M. Omoto y H. S. Kurtzman (Eds.). Orientación sexual y salud mental (pp. 131-150). American Psychological Association.

Colás, P. y Villaciervos, P. (2007). La interiorización de los estereotipos de género en jóvenes y adolescentes. Revista de Investigación Educativa, 25(1), 35-58.

Consejo Nacional para Prevenir la Discriminación (CONAPRED). (2018). Encuesta Nacional sobre Discriminación 2017: Prontuario de Resultado. Instituto Nacional de Estadística y Geografía (INEGI), Comisión Nacional de los Derechos Humanos (CNDH), Universidad Nacional Autónoma de México (UNAM), Consejo Nacional de Ciencia y Tecnología (CONACYT).

Corona, H. F. y Funes, D. F. (2015). Abordaje de la sexualidad en la adolescencia. Revista Médica Clínica Las Condes, 26(1), 74-80. doi: 10.1016/j.rmclc.2014.12.004

Corral, M. (2018). La violencia como práctica cotidiana. El caso de las juventudes LGBTI y su relación con las instituciones de derechos humanos en México. En R. Hernández y A. Winton (Coords.). Diversidad sexual, discriminación y violencia. Desafíos para los derechos humanos en México (pp. 57-66). Comisión Nacional de los Derechos Humanos.

Cubero, C. (21 de mayo de 2016). Tiene NL cuarto lugar en muertes por homofobia. Milenio. http://www.milenio.com/estados/tiene-nl-cuarto-lugar-en-muertes-por-homofobia

Deci,E.L.y Ryan, R.M.(2000).The "what" and “why" of goal pursuits:Human needs and the self-determination of behavior. Psychological Inquiry, 11, 227-268. https://doi.org/10.1207/S15327965PLI1104_01 
El rol de la edad en el estado de ánimo asociado al rechazo parental de la homosexualidad

De Lauretis, T. (2015). Género y teoría queer. Mora, 21(2),107-118. https://doi.org/10.34096\%2Fmora. $\mathrm{n} 21.2402$

De Bedout, A. (2008). El VIH-SIDA: Factores psicosociales y comportamentales. Revista de la Facultad de Ciencias de la Salud, 5(2), 129-140.

Elfakir, A. (2013). «Comment faire l'homme?» Sur la question de l'identité sexuelle dans un cas de psychose chez un homme. L'information psychiatrique, 6(89), 481-488.

Faul, F., Erdfelder, E., Lang, A.-G. y Buchner, A. (2007). G*Power 3: A flexible statistical power analysis program for the social, behavioral, and biomedical sciences. Behavior Research Methods, 39, 175-191. https://doi.org/10.3758/BF03193146

Fausto-Sterling, A. (2006). Cuerpos sexuados: La politica de género y la construcción de la sexualidad. Melusina.

Feinstein, B. A., Goldfried, M. R. y Davila J. (2012). The relationship between experiences of discrimination and mental health among lesbians and gay men: An examination of internalized homonegativity and rejection sensitivity as potential mechanisms. Journal of Consulting and Clinical Psychology, 80(5), 917-927. https://doi.org/10.1037/a0029425

Fernández, M. C.y Vázquez, F. (2013). En torno al rechazo, la salud mental y la resiliencia en un grupo de jóvenes universitarios gays, lesbianas y bisexuales. Revista Griot, 6(1), 44-65.

Flax, J. (1995). Psicoanálisis y feminismo. Pensamientos fragmentarios. Ediciones Cátedra.

Flores, G. y Solís, M. (2017). Terapia cognitivo-conductual y centrada en soluciones para la autoaceptación de la orientación sexual en un paciente homosexual. Revista Electrónica de Psicología Iztacala, 20(3), 1173-1202.

Forsell, S.L. (2017). New developments in LGBT development: what's new and what's (still) true. En R. Ruth y E. Santacruz (Eds.). LGBT psychology and mental health: emerging research and advances (pp. 15-46). Praeger.

Frommer, M.S. (2018). Offending gender - being and wanting in males same-sex desire. Studies in Gender and Sexuality, 1(2). https://doi.org/10.1080/15240650109349155

Frost, D. M. y Meyer, I. H. (2009). Internalized homophobia and relationship quality among lesbians, gay men and bisexuals. Journal of Counseling Psychology, 56, 97-109. https://doi.org/10.1037/ a0012844

Gil, G. E. (2007). El proceso de resiliencia en el desarrollo de la identidad lesbiana, gay y bisexual. Vector Plus, 30, 64-73.

Goffman, E. (2006). Estigma: la identidad deteriorada. Amorrortu Editores.

Goldberg, A. E. (2016). The Sage encyclopedia of LGBTQ studies. Sage.

Gómez, M. A. (6 de mayo de 2015). ¿Por qué es difícil saber cuántos homosexuales hay en el mundo? Dinero en Imagen. https://www.dineroenimagen.com/2015-05-06/55095\#view-2

Granados-Cosme, J. A. y Delgado-Sánchez, G. (2008). Identidad y riesgos para la salud mental de jóvenes gays en México: recreando la experiencia homosexual. Cadernos de Saúde Pública, 24(5), 1042-1050. 
Greene, K., Derlega, V. J. y Matthews, A. (2006). Self-disclosure in personal relationships. En A. L. Vangelisti y D. Perlman (Eds.). Cambridge handbook of personal relationships (pp. 409-427). Cambridge University Press.

Hatzenbuehler, M. L. (2009). How does sexual minority stigma "get under the skin"? A psychological mediation framework. Psychology Bulletin, 135, 707-730. https://doi.org/10.1037/a0016441

Hatzenbuehler, M. L., Nolen-Hoeksema, S. y Dovidio, J. (2009). How does stigma "get under the skin"?: The mediating role of emotion regulation. Psychologcal Science, 20(10), 1282-1289. https:// doi.org/10.1111/j.1467-9280.2009.02441.x

Hayes, A. (2018). Introduction to mediation, moderation, and conditional process analysis. Guilford.

Heatherington,L.y Lavner,J.A.(2008). Coming to terms with coming out: Review and recommendations for family systems-focused research. Journal of Family Psychology, 22(3), 329-343. https://doi. org/10.1037/0893-3200.22.3.329

Hébert, M., Fernet, M. y Blais, M. (2017). Le développement sexuel et psychosocial de l'enfant en de l'adolescent. Deboeck Supérieur.

Herek, G. M. (2000). The psychology of sexual prejudice. Current directions in psychological science, 9(1), 19-22. https://doi.org/10.1111/1467-8721.00051

Herek, G. M. (2004). Beyond "homophobia": Thinking about sexual prejudice and stigma in the twentyfirst century. Sexuality Research y Social Policy, 1(2), 6-24. https://doi.org/10.1525/srsp.2004.1.2.6

Herek, G. H. y Garnets, L. D. (2007). Sexual orientation and mental health. Annual Review of Clinical Psychology, 3, 353-375.

Hermosillo-De la Torre, A., Ángeles, V., Méndez, C., Palacios, P.y Sahagún, M. (2015). Sintomatología depresiva, desesperanza y recursos psicológicos: una relación con la tentativa de suicidio en una muestra de adolescentes mexicanos. Acta Universitaria, 25(449), 52-56. https://doi.org/10.15174/ au. 2015.900

Hernández, G. (2010). Los procesos holísticos de resiliencia en el desarrollo de identidades autorreferenciadas en lesbianas, gays y bisexuales (tesis doctoral). Universidad de Las Palmas de Gran Canaria, Departamento de Educación. http://bibaccedatest.ulpgc.es/bitstream/10553/4498/7/Tesis_ Resiliencia_LGB_Parte_I.pdf

Hinojosa, C. y Betancourt, A. (2007). En la cultura de la igualdad todas/os somos diversas/os. Preferencias sexuales y discriminación. CONAPRED.

Human Rights Watch. (2014). Not safe at home: Violence and discrimination against LGBT people in Jamaica. https://www.hrw.org/report/2014/10/21/not-safe-home/violence-and-discriminationagainst-lgbt-people-jamaica

Hunter, S. (2007). Coming out and disclosures: LGBT persons across the life span. Haworth.

Inda, N. (1996). Género masculino, número singular. En M. Burín y E. Dio Bleichmar (Comps.). Género, psicoanálisis, subjetividad (pp. 212-240). Paidós Mexicana.

Instituto Nacional de Estadística, Geografía e Informática (INEGI). (2018a). Encuesta Nacional sobre Discriminación (ENADIS). Principales resultados. https://www.conapred.org.mx/userfiles/files/ PtcionENADIS2017_08.pdf 
El rol de la edad en el estado de ánimo asociado al rechazo parental de la homosexualidad

Instituto Nacional de Estadística, Geografía e Informática (INEGI). (2018b). Encuesta Nacional sobre Discriminación (ENH). Principales resultados. https://www.inegi.org.mx/contenidos/programas/ enh/2017/doc/enh2017_resultados.pdf

Instituto Nacional de las Mujeres. (2009). Ley General de Acceso de la Mujeres a una Vida Libre de Violencia. Gobierno de México.

Jackson, S. D. y Mohr, J. J. (2016). Conceptualizing the closet: Differentiating stigma concealment and nondisclosure processes. Psychology of Sexual Orientation and Gender Diversity, 3(1), 80-92. https:// doi.org/10.1037/sgd0000147

Klein, K., Holtby, A., Cook, K. y Travers, R. (2015). Complicating the coming out narrative: becoming oneself in a heterosexist and cissexist world. Journal of Homosexuality, 62(3), 297-326. https://doi. org/10.1080/00918369.2014.970829

Kwon, P. (2013). Resilience in lesbian, gay, and bisexual individuals. Personality and Social Psychology Review, 17, 371-383. https://doi.org/10.1177/1088868313490248

Laqueur, T. (1994). La construcción del sexo: Cuerpo y género desde los griegos hasta Freud. Ediciones Cátedra.

Legate, N., Ryan, R. M. y Weinstein, N. (2012). Is coming out always a "good thing”? Exploring the relations of autonomy support, outness, and wellness for lesbian, gay, and bisexual individuals. Social Psychological and Personality Science, 3(2), 145-152. https://doi.org/10.1177/1948550611411929

Lehavot, K. y Simoni, J. M. (2011). The impact of minority stress on mental health and substance use among sexual minority women. Journal of Consulting and Clinical Psychology, 79(2), 159-170. https://doi.org/10.1037/a0022839

Lewis, R. J., Milletich, R. J., Derlega, V. J. y Padilla, M. A. (2014). Sexual minority stressors and psychological aggression in lesbian women's intimate relationships: The mediating roles of rumination and relationship satisfactions. Psychology of Women Quarterly, 38, 535-550. https://doi. org/10.1177/0361684313517866

Li, Y. y Samp, J. A. (2019). Internalized homophobia, language use, and relationship quality in same-sex romantic relationships. Communication Reports, 32(1), 15-28.

Lingiardi, V., Nardelli, N. y Drescher, J. (2015). New Italian lesbian, gay and bisexual psychotherapy guidelines: A review. International Review of Psychiatry, 27(5), 405-415. https://doi.org/10.3109/ 09540261.2015.1064875

Lipkin, A. (2018). Understanding homosexuality, changing schools (2a ed.). Routledge.

Lizárraga, X. (2014). Semánticas homosexuales. Reflexiones desde la antropología del comportamiento. INAH, México.

Lovibond, S. y Lovibond P. (1995). Manual for the depression, anxiety, stress scales. Psychology Foundation of Australia.

López, F. (2006). Homosexualidad y familia. Lo que los padres, madres, homosexuales y profesionales deben saber y hacer. Grao.

Lozano, I. y Salinas, F. (2016). Conociendo nuestra diversidad: discriminación, sexualidad, derechos, salud, familia y homofobia en la comunidad LGBTTTI. COPRED. 
Lozano, I., Fernández, J. A. y Baruch, R. (2017). Association between internalized homophobia and mental health indicators in LGBT individuals in Mexico City. Salud Mental, 40(5), 219226. https://dx.doi.org/10.17711/sm.0185-3325.2017.028

Malley, M.y Tasker, F. (2007). "The difference that makes a difference”: What matters to lesbians and gay men in psychotherapy. The Haworth Press, 11, 93-109. https://dx.doi.org/10.1300/J236v11n01_07

Maroto, A. L. (2006). Homosexualidad y trabajo social. Herramientas para la reflexión e intervención profesional. Siglo XXI Editores.

Marshal, M., Dietz, L., Friedman, M., Stall, R., Smith, H., McGinley, B., ... Brent, D. (2011). Suicidality and depression disparities between sexual minority and heterosexual youth: A meta-analytic review. Journal of Adolescent Health, 49, 115-123. https://doi.org/10.1016/j.jadohealth.2011.02.005

Meler, I. (2016). Cómo se construyen las relaciones de género en los sectores juveniles. Subjetividad y participación social. En M. L. Jiménez (Coord.). Jóvenes en movimiento en el mundo globalizado (pp. 93-114). Universidad Autónoma de México.

Melero, N. (2010). Reivindicar la igualdad de hombres y mujeres en la sociedad: Una aproximación al concepto de género. Barataria: Revista Castellano Manchega de Ciencias Sociales, 11, 73-83.

Mendoza, J. C., Ortiz, L.y Román, R. (2016). Principales resultados del Diagnóstico Situacional de Personas LGBTIQ de México 2015. UAM-Xochimilco.

Meyer,I. H. (1995). Minority stress and mental health in gay men. Journal of Health and Social Behavior, $36,38-56$.

Meyer, I. H. (2003). Prejudice, social stress, and mental health in lesbian, gay, and bisexual populations: Conceptual issues and research evidence. Psychological Bulletin, 129, 674-697. http://doi. org/10.1037/0033-2909.129.5.674

Mitchell, G. (2012). The development of psychoanalytic understandings of male homosexuality: Moving beyond pathology. Psycho-analytic Psychotherapy in South Africa, 20(1), 1-32.

Mohr,J.J. y Fassinger, R. (2003). Self-acceptance and self-disclosure of sexual orientation in lesbian, gay, and bisexual adults: An attachment perspective, Journal of Counseling Psychology, 50(4), 482-495. doi: 10.1037/0022-0167.50.4.482

Mosher, C. (2001). The social implications of sexual identity formation and the comingout process: A review of the theoretical and empirical literature. The Family Journal: Counseling and Therapy for Couples and Families, 9(2), 164-173. doi: 10.1177/1066480701092011

Mosteiro, M. J. y Porto, A. M. (2017). Análisis de los estereotipos de género en alumnado de formación profesional: Diferencias según sexo, edad y grado. Revista de Investigación Educativa, 35(1), 151-165. https://doi.org/10.6018/rie.35.1.257191

Organización Mundial de la Salud (OMS). (2018). Salud sexual. http://www.who.int/topics/sexual_ health/es/

Ortiz-Hernández,L. (2005). Influencia de la opresión internalizada sobre la salud mental de bisexuales, lesbianas y homosexuales de la Ciudad de México. Salud Mental, 28(4), 49-65.

Osborne, R. y Molina, C. (2008). Evolución del concepto de género. EMPIRIA. Revista de Metodología de Ciencias Sociales, 15, 147-182. 
El rol de la edad en el estado de ánimo asociado al rechazo parental de la homosexualidad

Paul, J. P., Catania, J., Pollack, L., Moskowitz, J., Canchola, J., Mills, T. ... Stall, R. (2002). Suicide attempts among gay and bisexual men: Lifetime prevalence and antecedents. American Journal of Public Health, 92, 1338-1345. https://doi.org/10.2105/AJPH.92.8.1338

Pichardo, J. I. (2012). El estigma hacia personas lesbianas, gays, bisexuales y transexuales. En E. Gaviria, C. García-Ael y F. Molero (Coords.). Investigación-acción. Aportaciones de la investigación a la reducción del estigma (pp. 111-125). Sanz y Torres.

Piedra, J., Rodríguez, A. R., Ries, F. y Ramírez, F. G. (2013). Homofobia, heterosexismo y educación física: Percepciones del alumnado. Profesorado, 17(1), 325-338.

Pineda, G. (25 de enero de 2018). Tras 80 días de plantón LGBT, se abre el clóset en el Congreso de Nuevo León. CCNerws. https://news.culturacolectiva.com/derechos-humanos/colectivos-lgbtven-posible-iniciativa-de-matrimonio-igualitario-en-nuevo-leon/

Planned Parenthood. (2018). Sexual orientation. Sexual orientation and gender. https://www. plannedparenthood.org/learn/sexual-orientation-gender/sexual-orientation

Posner, K., Brodsky, B., Yershova, K., Buchanan, J. y Mann, J. (2014). The Oxford handbook of suicide and self-injury. Oxford University Press.

Quintana, A. y Vásquez, E. (2003). Construcción social de la sexualidad adolescente: Género y salud sexual. IES.

Richardson, E. M. (2004) 'A ripple in the pond': Challenging homophobia in a teacher education course. Education as Change, 8(1), 146-163. https://doi.org/10.1080/16823200409487084

Rocha, T. E. y Díaz-Loving, R. (2011). Identidades de género: más allá de mitos y cuerpos. Trillas.

Russell, S.T. (2006). Salud mental, abuso y consumo de sustancias entre los jóvenes de minorías sexuales: evidencia del estudio Add Health. En A. M. Omoto y H. S. Kurtzman (Eds.). Orientación sexual y salud mental (pp. 13-33). American Psychological Association.

Russell, G. M.y Richards, J.A. (2003). Stressor and resilience factors for lesbians, gaymen, and bisexuals confronting antigay politics. American Journal of Community Psychology, 31, 313-328. https://doi. org/10.1023/a:1023919022811

Ryan, C. (2009). Niños saludables con el apoyo familiar. Ayuda para familias con hijos e hijas lesbianas, gays, bisexuales y transgénero. Marian Wright Edelman Institute, San Francisco State University.

Ryan, C., Huebner, D., Diaz, R. M. y Sanchez, J. (2009). Family rejection as a predictor of negative health outcomes in white and Latino lesbian, gay, and bisexual young adults. Pediatrics, 123(1), 346-352. https://doi.org/10.1542/peds.2007-3524

Ryan, R. M. y Deci, E. L. (2000). Self-determination theory and the facilitation of intrinsic motivation, social development, and well-being.American Psychologist, 55, 68-78. https://doi.org/10.1037/0003066X.55.1.68

Ryan, W. C., Legate, N. y Weinstein, N. (2015). Coming out as lesbian, gay, or bisexual: The lasting impact of initial disclosure experiences. Self and Identity, 14(5), 549-569. https://doi.org/10.1080/ 15298868.2015.1029516

Saeteros, R., Sanabria, G. y Pérez, J. (2014). Imaginario sobre la homosexualidad en estudiantes de politécnicos ecuatorianos. Revista Cubana de Salud Pública, 40(4), 299-313.

Savin-Williams,R.C.y Diamond,L.M.(2000).Sexualidentity trajectories among sexual-minority youths: gender comparisons. Archives of Sexual Behavior, 29(6), 607-627. doi: 10.1023/a:1002058505138 
Secretaría de Salud. (2017). Protocolo para el acceso sin discriminación a la prestación de servicios de atención médica de las personas lésbico, gay, bisexual, transexual, travesti, transgénero e intersexual y guias de atención especificas. Institutos Nacionales de Salud Hospitales de Alta Especialidad.

Shively, M. G. y De Cecco, J. P. (1977). Components of sexual identity. Journal of homosexuality, 3(1), 41-48. doi: 10.1300/J082v03n01_04

Simpson, C. C., Sutter, M. y Perrin, P. B. (2016). Can community consciousness be a bad thing? A moderated mediation analysis of heterosexism, mental health and body appreciation in sexual minority men. Culture, Health E Sexuality, 18(11), 1279-1294. https://doi.org/10.10 80/13691058.2016.1183047

Smith, D. E. (2018). Homophobic and transphobic violence against youth: The Jamaican context. International Journal of Adolescence and Youth, 23(2), 250-258. https://doi.org/10.1080/02673843 .2017 .1336106

Stolcke, V. (2004). La mujer es puro cuento: La cultura del género. Estudios Feministas, Florianópolis, 12(2), 77-105.

Szymanski, D. M., Ikizler, A. S. y Dunn, T. L. (2016). Sexual minority women's relationship quality: Examining the roles of multiple oppressions and silencing the self. Psychology of Sexual Orientation and Gender Diversity, 3, 1-10. https://doi.org/10.1037/sgd0000145

United Nations Educational, Scientific and Cultural Organization (UNESCO). (2016). Out in the open: Education sector responses to violence based on sexual orientation and gender identity/expression. UNESCO.

Varas-Díaz, N., Serrano-García, I. y Toro-Alfonzo, J. (2004). Estigma y diferencia social: VIH/SIDA en Puerto Rico. Ediciones Huracanes.

Vela, E. (2017). La discriminación en el empleo en México. Instituto Belisario Domínguez, Senado de la República.

Vera, R. (1 de agosto de 2018). En lo que va de 2018 se han cometido 26 crímenes de odio por homofobia. Proceso. https://www.proceso.com.mx/545299/en-lo-que-va-de-2018-se-hancometido-26-crimenes-de-odio-por-homofobia

Verrelli, F., White, F. A., Harvey, L. J. y Pulciani, M. R. (2019). Minority stress, social support, and the mental health of lesbian, gay, and bisexual Australians during the Australian Marriage Law Postal Survey. Australian Psychology, 54(4), 336-346. https://doi.org/10.1111/ap.12380

Vespucci, G. (2017). Homosexualidad, familia y reivindicaciones: De la liberación sexual al matrimonio igualitario. Unsam-Edita.

Warner, M. (1993). Fear of a queer planet: Queer politics and social theory. University of Minnesota Press.

White, Y., Sandfort, T., Morgan, K., Carpenter, K. y Pierre, R. (2016). Family relationships and sexual orientation disclosure to family by gay and bisexual men in Jamaica. International Journal of Sexual Health, 28(4), 306-317. https://doi.org/10.1080/19317611.2016.1227015

Wittig, M. (2006). El pensamiento heterosexual y otros ensayos. Egales.

Zambrano, C., Ceballos, A. y Ojeada, D. (2017). Reconocimiento de la orientación homosexual. Revista Psicoespacios, 11(19), 77-93. https://doi.org/10.25057/21452776.940

Zupančič, A. (2017). What is sex? MIT Press. 